\title{
Origin of intramuscular nerve action potential
}

\author{
LUDWIG GUTMANN, * HANNS C HOPF, $†$ ROLF ROEDER $\dagger$ \\ From the Departments of Neurology, West Virginia University School of Medicine, Morgantown, WV, USA* \\ and University of Mainz, Mainz, Federal Republic of Germany $\dagger$
}

SUMMARY The origin of the intramuscular nerve action potential (INAP) was investigated using conventional surface recording electrodes. The appearance of the INAP was (1) associated with subjective paresthesias, (2) recorded only when the reference electrode (G2) was over an appropriate digital nerve, (3) increased in latency as G2 was placed more distally, (4) blocked by an orthodromic sensory nerve action potential arising from the thumb, and (5) abolished with an anaesthetic block in the digital nerve proximal to G2. The findings indicate that the INAP is recorded by the G2 electrode from the digital nerve.

The muscle action potential (MAP) evoked by supramaximal median or ulnar nerve stimulation is preceded by a separate small potential-the intramuscular nerve action potential (INAP). Initially thought to arise from motor axons, ${ }^{1}$ later studies indicated that it originated from sensory axons. ${ }^{23}$ The INAP is seen only at high gain amplification. Its importance relates to it being confused as a MAP in patients with disorders selectively affecting peripheral motor axons (such as motoneuron disease, brachial plexus root avulsion). ${ }^{3}$ Using conventional surface recording electrodes our studies indicate that the INAP arises from sensory axons in digital nerves.

\section{Materials and methods}

Studies were performed on three normal (authors) and five patient controls. Median and ulnar nerves or their branches were stimulated at the wrist percutaneously or with stainless steel insect pin electrodes placed $20 \mathrm{~mm}$ apart. Stimulation of the thumb during double stimulation was with two cleaners placed $20-30 \mathrm{~mm}$ apart.

Muscle and nerve action potentials were recorded with surface disc electrodes. At the beginning of each study the recording electrode (G1) was placed over motor point of thenar or hypothenar muscles and the reference electrode (G2) laterally over the tendon of the thumb and fifth digit (5-10 $\mathrm{mm}$ distal to the metacarpo-phalangeal joint) stimulating median and ulnar nerves respectively at the wrist. In several studies the recording and/or reference electrode locations were varied. Digital nerve action potentials

Address for reprint requests: Dr Gutmann, Department of Neurology, West Virginia University School of Medicine, Morgantown, WV 26506, USA

Received 3 February 1987 and in revised form 20 May 1987. Accepted 22 May 1987
(DNAP) were recorded from lateral surface of thumb placing G1 (proximal) and G2 $20 \mathrm{~mm}$ apart. Mepivacaine hydrochloride (Scandicain) and lidocaine (Xylocaine) were used to anaesthetise locally the lateral digital nerve of the thumb at the metacarpo-phalangeal joint proximal to $G 2$ on the thumb. Each segment of the study was carried out on at least two individuals.

\section{Results}

Median or ulnar nerve stimulation at the wrist in all eight subjects evoked a MAP that was always preceded by INAP when Gl was over the motor point of the muscle and G2 5-10 mm distal to the metacarpalphalangeal joint of the thumb and fifth finger respectively. Adjusting the intensity in each trial allowed a submaximal stimulus to evoke the INAP without a subsequent MAP (fig la). This was accompanied by the sensation of paraesthesia in the median or ulnar sensory distribution in all eight subjects.

Stimulating median nerve submaximally evoked a simultaneous INAP and DNAP in three subjects. Both were larger with supramaximal nerve stimulation (fig la).

Median nerve stimulation in two subjects was performed placing $\mathrm{G} 2$ over the dorsum of first metacarpal bone (G1 over thenar muscle). No INAP was evoked (fig 1b). Stimulation of ulnar nerve in these two subjects recording simultaneously from hypothenar (G2 lateral fifth finger) and first dorsal interosseous muscles (G2 lateral surface index finger) evoked a INAP from the former but not the latter (fig lc).

Stimulating the median nerve at the wrist, the INAP latency was relatively short (peak latency 


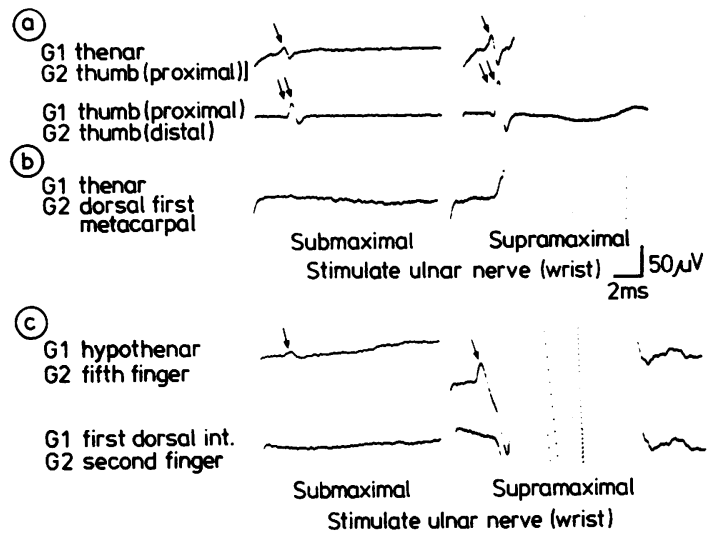

Fig 1 (a) INAP (arrow) and DNAP (double arrow) evoked simultaneously on submaximal and supramaximal median nerve stimulation at the wrist. (b) No INAP evoked on median nerve stimulation with $G 2$ over first metacarpal bone (Gl over thenar muscle). (c) INAP (arrow) evoked on ulnar nerve stimulation with $G 2$ over digital nerve of fifth finger (Gl over hypothenar muscle) but not when over digital nerve of second finger (Gl over first dorsal interosseus muscle).

$2.7 \mathrm{~ms}$ ) with short conduction distance (G2 just distal to metacarpo-phalangeal joint $35 \mathrm{~mm}$ distal to $\mathrm{G} 1$ ). The latency increased (peak latency $3.0 \mathrm{~ms}$ ) with longer distance (G2 just distal to interphalangeal joint $72 \mathrm{~mm}$ distal to $\mathrm{G} 1$ ).

Double stimulation (collision technique) using supramaximal stimuli of the digital nerve of the thumb (initial stimulus) and median nerve at the wrist (second stimulus) was performed in two subjects recording from thenar muscle $(\mathrm{Gl})$ and lateral thumb (G2), the latter proximal to digital nerve stimulating electrodes. The INAP maximal amplitude was at interstimulus intervals greater than $6 \mathrm{~ms}$ and the smallest amplitude at $3 \mathrm{~ms}$ interval (fig 2a).

Following anaesthetic block of digital nerve of thumb proximal to G2 in two subjects, INAP was no longer recorded when stimulating the median nerve at wrist with submaximal $(3.6 \mathrm{~mA})$ and supramaximal $(10 \mathrm{~mA})$ stimuli (fig $2 \mathrm{~b})$.

\section{Discussion}

The origin of the INAP that precedes the thenar and hypothenar MAP arises from sensory axons. ${ }^{23}$ The INAP can be evoked at lower stimulation thresholds than MAP suggesting the INAP arises from large sensory afferents (for example muscle spindles and/or tendon organs). Fiaschi suggested the INAP arose from the palmar segment of large sensory axons innervating the thumb, utilising concentric recording electrodes in the thenar muscle. ${ }^{2}$

Our studies further define the origin of the INAP (a)

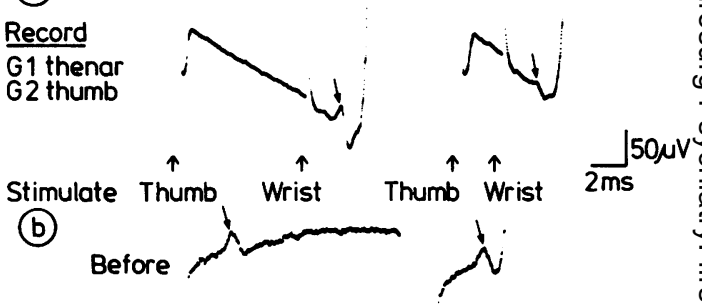

Scandicain

After

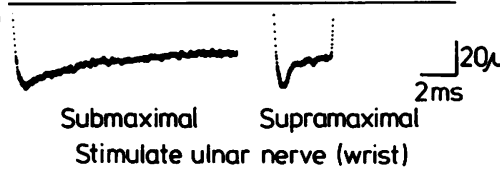

Record G1 thenar

G2 thumb (prox.)

Fig 2 (a) Collision technique stimulating digital nerve of thumb and median nerve at wrist. The INAP (arrow) is much smaller at interstimulus time of $2.8 \mathrm{~ms}$ than $7.6 \mathrm{~ms}$. G1 over thenar muscle; $G 2$ over digital nerve of thumb. (b) INAP (arrow) evoked on median nerve stimulation disappeared after scandician infiltration of digital nerve.

utilising more conventional surface recordings who G1 placed over the motor point of the muscle and G2 distally over the finger. The INAP and DNAPwere consistently evoked with submaximal netro stimulation at a threshold below that of the MAP agod associated with subjective paraesthesias implying 胃 INAP in part comes from cutaneous afferent fib with a low stimulation threshold.

When $G 2$ is placed over areas in which (1) digitabo nerve is a branch of a nerve other than the one being stimulated or (2) no digital nerve is present, no INAP occurs. Utilising the collision technique, the INAP ors median nerve stimulation can be blocked by an orthodromic sensory nerve action potential arising fronf the thumb. The INAP latency increases with location of the $\mathrm{G} 2$ more distally on the digit. Lastly, the INAPstimulating median nerve is abolished by an anaes 3 thetic block in the digital nerve just proximal to G2J All these observations indicate that the INAP (1 arises from the digital nerve of the thumb and fifth fingers in the case of median and ulnar nerve stimu lation respectively and (2) is recorded by the reference electrode lying over the digital nerve.

\section{References}

1 Buchthal F, Rosenfalck A. The intramuscular nerve action potential. Brain Res 1966;3:72-73.

2 Fiaschi A. Observations on the sensory nature of the intramuscular nerve action potential. J Neurol Neuro $\overline{\bar{N}}$ surg Psychiatry 1973;36:509-13.

3 Gutmann $L$. The intramuscular nerve action potential J Neurol Neurosurg Psychiatry 1969;32:193-6. 